
\title{
25 Research Soure \\ Horizontally ridge reconstruction using titanium mesh with tenting screw vs autologous bone graft: a retrospective study
}

\section{Lin Zhou}

Fujian Medical University

\section{Yifeng Xing}

Fujian Medical University

Jiamin Ding

Mucosa Affiliated Stomatological Hospital of Fujian Medical University

Yanjun Lin

Fujian Medical University

Chaowei Liu

Fujian Medical University

Jiang Chen

Fujian Medical University

Dong Wu ( $\nabla$ wudong@fjmu.edu.cn )

Fujian Medical University

\section{Article}

Keywords: Titanium mesh, Tenting screw, Autogenous bone, Guided bone generation, Reference plane, Exposure

Posted Date: June 29th, 2022

DOI: https://doi.org/10.21203/rs.3.rs-1175632/v2

License: (a) (1) This work is licensed under a Creative Commons Attribution 4.0 International License. Read Full License 


\section{Abstract}

The aim of this retrospective study was to evaluate the horizontal bone gain at the different level of alveolar bone using titanium screw as a reference plane by titanium mesh with tent screw (exposed or not) and autologous bone grafting. 16 patients and 23 measured points were enrolled in this study. CBCT data of immediately and 6 months after bone grafting were measured at crestal (CW), midway (MW), and basal (BW) level and reconstructed for area measurement. The exposure of Ti-mesh or not had been evaluated. The area of bone gained and absorption ratio were almost the same. The width absorption ratio of titanium mesh and onlay grafting group or at CW, MW, and BW were almost the same. However, the width absorption ratio of the exposed Ti-mesh at $\mathrm{CW}, \mathrm{MW}$, and $\mathrm{BW}$ were significantly higher than the not exposed one. Thin biotype was a main factor of titanium mesh exposure. The exposed Ti-mesh at late phase also can achieve clinically accepted bone gained after certain anti-inflammatory treatment. Timesh with tenting screw could achieve the same bone augmentation effect as the autogenous bone block did, even it is exposed at late phase. However, the absorption ratio significantly increased if exposed.

\section{Introduction}

Severe bone atrophy in the implant area especially in the anterior region is difficult and challenge for dental implant, which may affect the initial stability of the implant and the morphology of the soft tissue[1]. There are many surgical techniques to treat the compromised alveolus in the anterior dental implant area, including the application of small-diameter implants, autologous bone block grafting, bone splitting and guided bone regeneration techniques[2]. Autologous bone grafting had been regarded as the gold standard for bone grafting due to its good osteoconductivity, osteoinductivity and biocompatibility[3, 4]. The source of autogenous bone is generally taken from the chin or the external oblique line of the mandible which would brought the second surgical area and may increasing the probability of infection and the pain $[5,6]$, it is often not easy to be accepted by patients especially in China which also limits its clinical application.

Guided bone regeneration (GBR) is considered to be the best method in the reconstruction of bone defects around implants, and makes the new bone formation predictable and long-term stability[7]. There are four principles of GBR: Primary healing, Ascularization, Space, and Satbility[8]. The osteogenesis space maintained by the barrier membrane and the stability of this space both play an important role in the success of GBR[9]. According to whether it can be absorbed in the body, barrier membranes are mainly divided into two types: absorbable (such as collagen membrane, etc.) and non-absorbable (such as titanium mesh, etc.)[10]. Studies have reported that the use of titanium mesh as a barrier membrane for GBR can have good osteogenesis due to its maintain and stability of the osteogenesis space, but there are complications such as wound dehiscence and exposure of titanium mesh[11].

Titanium mesh could use as a non-absorbable barrier membrane for horizontal and vertical bone regeneration, the mean achieved bone amount was $4.91 \mathrm{~mm}$ (range: $2.56-8.6$ ) for vertical reconstruction, 
while a mean of $4.36 \mathrm{~mm}$ (range: 3.75-5.65) for horizontal reconstruction[11]. The measurement points of many research of horizontal augmentation lacked a certain reference point, and the measurement points were mainly concentrated on the top of alveolar ridge ignoring the base of alveolar bone which played a significant role in the primary stability of secondly implant placement. The exposure of titanium mesh at different phase are the main complication of this technique which incidence range from $5-50 \%$ [12], and how is the bone augmentation after the exposure of titanium mesh was rarely reported.

Tenting screws as pillars can provide good and support for the osteogenesis space, combined with titanium mesh. As a rigid support structure, tenting screws can prop up the titanium mesh to resist the pressure of the lips. A tenting screw combined with titanium mesh may reduce the need for autogenous bone grafting in the reconstruction of severely atrophic ridges, and improves the survival of implants[13, 14]. The gained bone width was 0.91 to $4.37 \mathrm{~mm}$ in grafting with tenting screws[15]. But the related research still rare.

Therefore, the aim of this retrospective study was to evaluate the horizontal bone gain at the crestal, midway and basal level of alveolar bone as Block et al mentioned[16] using titanium mesh with tenting screw vs autologous bone grafting of horizontally deficient maxillary ridges with class 4 of Benic\&Hammerl alveolar ridge bone defect classification[17]. Moreover, we compared the gained bone where the titanium mesh is exposed with the titanium mesh is not exposed.

\section{Results}

\section{Patient profiles}

In the present retrospective study, 9 patients ( 3 male and 6 female) with 14 tent screws in titanium mesh group and 7 patients ( 3 male and 4 female) with 11 titanium screws in autologous bone group were enrolled. The detail of the mean age, the region of bone defect, smoking, periodontal status and gingival biotype were listed in Table 1.

\section{Complication}

The exposure was occurred in 6 titanium mesh of 4 patients. One exposure of the titanium mesh in one patient was occurred at two weeks after surgery, and the dehiscence of soft tissue continues to be widen after alternately rinsed with hydrogen peroxide and normal saline each three day and treated with topical minocycline hydrochloride ointment for 2 weeks. Therefore, the titanium mesh was removed at 4 weeks after surgery and the grafted bone was tofukasu-like, and it was defined as failure.

The other 5 exposure of titanium mesh happened in 3 patients at the late stage, including two side of the anterior maxillary region in two patients, one side of the anterior maxillary region in one patients. Among them one small dehiscence was healed after using the above treatment, while the other four maintain the size of the crack without inflammation at each two weeks follow-up. These four were defined as exposure. 
It is worth noting that patients with thin gingival biotype underwent the exposure of titanium mesh, and the dehiscence of the soft tissues are all at the sharp edges of the titanium mesh. After trimming the edges of the titanium mesh and rinsing to reduce inflammation, the soft tissue healed in the thick gingival biotype patient and the other maintain the size of the dehiscence without inflammation in the thin gingival biotype patients.

\section{Radiographic outcome}

1) The area of the augmented bone

The mean area of the augmented bone at the cross section of the titanium screw in onlay grafting group after 6 months of surgery was $(49.20 \pm 11.29) \mathrm{mm}^{2}$ and the absorption rate was $(25.52 \pm 0.41) \%$.

Meanwhile, the mean area of the augmented bone at the cross section of the tent screw in titanium mesh group after 6 months of surgery was $(46.97 \pm 22.02) \mathrm{mm}^{2}$ and the absorption ratio was $(24.66 \pm 16.04) \%$. These demonstrated that the average bone gain area, which meet the basic requirement for implant placement, at the reference plane was almost the same without statistical different as shown in Fig 1A. And the area absorption ratio was also almost the same as shown in Fig 1B.

2) The width of the augmented bone

The gained bone width after 6 months of surgery in both groups at different measured level were showed in Table 2. The gained bone width at titanium mesh group was divided into exposure group and not exposure one. Although there were not statistical different between the titanium mesh and onlay grafting about the gained bone width at CW, MW, and BW after 6 months of surgery. But from the data point of view, the width at $\mathrm{CW}, \mathrm{MW}$, and $\mathrm{BW}$ of augmented bone of titanium mesh seem higher than the onlay grafting group. And the gained bone width at $\mathrm{CW}, \mathrm{MW}$, and BW were almost the same no matter in titanium mesh or onlay grafting group. There were not statistical different between the titanium mesh exposure group and not exposure one about the gained bone width at CW, MW, and BW after 6 months of surgery. But the gain width of not exposure group seem higher than the exposure group from the data point of view. These results were showed in Fig 2.

There were not statistical different between the titanium mesh and onlay grafting about the width absorption ratio at $\mathrm{CW}, \mathrm{MW}$, and BW after 6 months of surgery. Meanwhile the width absorption ratio at $\mathrm{CW}, \mathrm{MW}$, and $\mathrm{BW}$ were almost the same no matter in titanium mesh or onlay grafting group. However, the width absorption ratio of the titanium mesh exposure group at $\mathrm{CW}, \mathrm{MW}$, and $\mathrm{BW}$ were significantly higher than the not exposure one as it is showed in Fig 3.

\section{Discussion}

In the present retrospective study, the horizontal ridge augmentation of titanium mesh in 14 bone graft sites of 9 patients were compared with autologous bone in 11 bone graft sites of 7 patients. The reason why using autologous bone block as a comparison is that it had been used for a long time with an 
almost $97.1 \%$ success rate and regard as the gold standard for horizontal and vertical ridge augmentation[18]. Both the technique of titanium mesh with tenting screw and autologous bone on-lay grafting can achieve clinically acceptable effect execpt one failure case, which allowed implant placement after 6 months of surgery with or without further GBR.

There are many treatment modalities for management of severely thin alveolar ridges, including titanium mesh, onlay grafting and sausage technique by Urban. Many of these technologies are using the principle of GBR, especially the role of the barrier membrane to stabilize the osteogenesis space. The titanium mesh with a macroporous structure was used in our present study. The macroporous structure of titanium mesh was a double-edged sword, it can provide a better blood supply by promoting the growth of new blood vessels on the one hand, on the other hand the soft tissue cells can also enter the osteogenic area through the pores and bring a negative impact[19].

The area of the augmented bone in titanium mesh after 6 months of surgery was almost the same as the onlay grafting group, and the augmented bone width at CW, MW, and BW were the same with the onlay grafting no matter whether the titanium mesh was exposure or not. These demonstrated that the osteogenesis effect of titanium mesh is equivalent to that of autogenous bone, and seemed even better if the titanium mesh was not exposure which was derived from the data point of view.

In the present study, we measured the gained bone at three different levels which were crestal, midway, and basal level. The significance of this measurement is that it can distinguish the effect of bone augmentation at different bone level[20]. The bone width at basal and midway level can make sure whether the implant placement can get enough primary stability, while the bone width at crestal level allows us to predict whether the implant placement needs further GBR. The bone gain at the three level were almost the same at onlay grafting and titanium mesh groups. These demonstrated that the titanium mesh can maintain the space at these three levels well by resisting the pressure of lip. The bone gain ranged from $2.0 \mathrm{~mm}$ to $9.7 \mathrm{~mm}$ at different level in titanium mesh group, which was consistent with other related research[21, 22].

The absorptivity of the titanium mesh at different levels were almost the same with the onlay grafting. But the average bone gain at different level of titanium mesh was seem higher than the onlay grafting from the data point of view. This may be due to the limited thickness of the autogenous bone, especially the thickness of the cortical bone[23]. However, when using titanium mesh, the amount of xenogeneic bone can be increased appropriately to prevent a relatively large probability of absorption[24].

When the titanium mesh is exposed, its absorption ratio is significantly higher than not exposed. The late exposure may result in graft absorption of approximately $15 \%-25 \%$ of the exposed area, resulting in a slight bone deficiency at the exposed site[25].

The failure rate of titanium mesh was $7.14 \%$, but the exposure rate of the titanium mesh was $33.33 \%$ in patients and $35.71 \%$ in bone graft sites. The incidence of titanium mesh exposure was similar to other literatures, as Cai et al reported in her review that the rate of the exposure was mostly $20-30 \%$ and the 
highest reported exposure rate was $66 \%[26]$. One titanium mesh exposure at early stage with inflammation and continuous lager dehiscence, and the titanium mesh was removed in our study. Some researcher reported that the titanium mesh should be removed as soon as possible if the exposure occurred at early stage, because the increased fibrous tissue may damage the osteogenesis of residual xenograft particles and resulted in decrease in bone formation[27]. In the present study, the residual xenograft particles were tofukasu-like after remove the mesh, it may be effect by the fibrous tissue and the continuous inflammation. There were 5 titanium mesh exposure at the late stage, one small dehiscence healed and the other four maintain the size of the crack after anti-infection treatment. The reason why the titanium mesh did not need to removed was that the higher surface smoothness reduced the adsorption and reproduction of bacteria when the mesh was exposure[28].

It is worth noting that the exposure of titanium mesh is closely related to gingival biotype. One clear important factor behind exposure of the titanium mesh was thin soft tissue biotype, because it was less resistant to trauma and had a compromised vascular network[29]. Four patients were thin gingival biotype in the present study, and unfortunately these four patients all underwent the exposure of titanium mesh. There was still a certain amount of tissue tension even after adequate periosteal scoring and tension free closure[30], the dehiscence of the soft tissue is more likely happened in the patients with thin gingival biotype especially at the edge of the titanium mesh.

The limitation of the present study was that the sample size was not enough as a retrospective study. There was selection bias in the present study as a retrospective study. Thought the measurement was done by one dentist, the subjective bias was still inevitable.

\section{Methods}

\section{Patient selection}

From April 2018 to January 2020, patients who need bone augmentation before implant placement were collected from department of oral and maxillofacial implant research center, Affiliated Stomatological Hospital of Fujian Medical University. All patients were informed about the surgical and restoration treatment procedure. The study design was performed in accordance with the Helsinki Declaration (revised in 2008).

Inclusion criteria:

1) Class 4 of Benic\&Hammerl alveolar ridge bone defect classification in anterior region. 2) Bone augmentation with titanium mesh with tenting screw or autologous bone grafting before implant placement. 3) Good systemic and oral health

Exclusion criteria:

1) Systemic or local acute inflammation. 2) Patients who received radiotherapy or chemotherapy within the last 5 years. 3) Smoker (more than 15 cigarettes/day) or alcohol abuse. 4) Patients who cannot 
tolerate implant surgery. 5) Long-term use of immunosuppressive agents or double Phosphonate drugs. 6) Uncontrolled diabetic patients (fasting blood glucose $\geq 8.88 \mathrm{mmol} / \mathrm{L}$ )

\section{Preoperative preparation}

History of systemic system diseases, infectious diseases and drug allergies were inquired and cone beam computer tomography (CBCT) examination was performed to evaluate whether the alveolar ridge bone defect belong to Class 4 of Benic\&Hammerl classification. All patients received periodontal cleansing 1 week before surgery, and antibiotics were used prophylactically 3 days before surgery.

\section{GBR with titanium mesh}

All the surgeries were performed by an experienced surgeon. A full-thickness mucoperiosteal flap was reflected with two vertical incisions in the adjacent teeth under local anesthesia with Primacaine ${ }^{\circledR}(4 \%$ Articaine,1/100000 adrenaline, ACTEON). Nourishing holes were prepared on the surface of the cortical bone with small ball drill in order to guarantee the blood supply. A tenting screw (Neo biotech) was fixed onto the alveolar ridge after the nourishing holes were prepared. Selecting an appropriate size titanium mesh (SMARTbuilder ${ }^{\mathrm{TM}}$ OSSTEM), and trim and pre-bend the titanium mesh according to the shape of the alveolar ridge. Xenogeneic bone (Geistlich Bio-Oss) was used to fill the space between the bone surface and titanium mesh. Then the titanium mesh was fixed to the tenting screw with corresponding screw. Finally, the titanium mesh surface was covered by an absorbable biomembrane (Geistlich BioGide). The sub-periosteal incisions were also made in order to achieve primary closure, then the flap was repositioned and sutured. The briefly process was showed in figure $4 \mathrm{~A}-\mathrm{E}$.

\section{Onlay grafting with autologous bone block}

The exposure of the alveolar ridge was using the same surgery protocol with the titanium mesh group. The autologous bone was collected from mandibular external oblique line or chin. The autologous bone block was prepared by piezosurgery at donor site according to the size of the bone defect area measured by $\mathrm{CBCT}$ before operation. The autologous bone block was fixed on the alveolar ridge by using a titanium nail. The gap was filled with xenogeneic bone (Geistlich Bio-Oss), and the recipient site was covered by an absorbable biomembrane (Geistlich Bio-Gide). The sub-periosteal incisions were made in order to achieve primary closure, then the flap was repositioned and sutured. The briefly process was showed in figure 4FJ.

\section{Secondly implant placement}

The implant placement protocol was made according to the CBCT images, which was taken 6 months after the bone grafting operation. The process of implant placement was according to the manufacturer's instructions. Whether the GBR needed to be done was depended on whether there was enough bone around the implant neck. The restoration process was done at 3 or 4 months after implant placement.

\section{Outcome measurements}




\subsection{Complications}

1) the exposure of titanium mesh at early stage

The exposure of titanium mesh happened within first month after surgery was define as early stage. The titanium mesh should be removed if the soft tissue dehiscence continues to be widen after alternately rinsed with hydrogen peroxide and normal saline each three day and treated with topical minocycline hydrochloride ointment for 2 weeks. These cases were defined as failure. If the soft tissue healing after related treatment, it was still defined as success.

2) the exposure of titanium mesh at late stage

The exposure of titanium mesh happened after first month of surgery was define as later stage. If the soft tissue dehiscence did not continue to be widen and no inflammation occured after treatment mentioned above, the patient should be followed in every week, and these cases were defined as exposure. If the soft tissue dehiscence continued to be widen, the titanium mesh should be removed, and it was defined as failure.

3 the exposure of autogenous bone block or titanium screw

If the autogenous bone block exposure and the soft tissue can't heal after treatment mentioned above or there was continuous soft tissue inflammation, the grafted bone need to be removed, which should define as failure.

\subsection{Radiographic evaluations}

The CBCT imaging data of each patient were collected before bone grafting (T0), immediately after bone grafting (T1), and 6 months after bone grafting (T2). All CBCT scans were performed in the same radiology centre using the same parameters (NewTom, Italy with voxel size $0.150 \mathrm{~mm}$, tube voltage of 90 $\mathrm{kV}$, current of $7.00 \mathrm{~mA}$, and exposure time of $9 \mathrm{~s}$ ), and all measurements were taken by the same examiner.

1) the area of the augmented bone

The CBCT DICOM data of T1 and T2 was imported into Mimics Medical 21.0 software. The cross section of the titanium screw (onlay grafting group) or tenting screw (titanium mesh group) was taken as the reference plane. The labial bone was reconstructed in the software immediately and 6 months after the operation according to the same reference plane as shown in Fig 5.

2) the width of the augmented bone

The width of three different level, which were crestal width (CW), midway width (MW), and basal width (BW), were measured according to the Block et al method using the titanium nail (onlay grafting group) or tent screw (titanium mesh group) as the reference plane at T0, T1, and T2 time period. Therefore, the 
increase horizontal bone width was: $\mathrm{W}_{\mathrm{T} 2}-\mathrm{W}_{\mathrm{T} 0}$, the absorption of bone width was: $\mathrm{W}_{\mathrm{T} 1}-\mathrm{W}_{\mathrm{T} 2}$, and the absorption rate of bone width was $\left(\mathrm{W}_{\mathrm{T} 1}-\mathrm{W}_{\mathrm{T} 2}\right) /\left(\mathrm{W}_{\mathrm{T} 1}-\mathrm{W}_{\mathrm{T} 0}\right) * 100 \%$ at 6 months after surgery. The measured points at different level was showed in Fig 6 . The detail measured method are as follow: A point is the concavity point on the buccal cortex, $\mathrm{M}$ point is the midpoint of the crestal alveolar ridge, $\mathrm{MN}$ line is the line pasting point $M$ and paralleling lingual cortex, $A B, E F$, and $C D$ line are the lines vertical to $M N$ at alveolar basal bone, alveolar crestal bone, and alveolar midway bone. The distances between $A B, C D$ and $\mathrm{EF}$ are equal, parallel to each other and perpendicular to $\mathrm{MN}$, and the $\mathrm{T} 1, \mathrm{~T} 2$ period measurement method is similar to T0.

\section{Statistical analysis}

The data of the width absorption ratio, the area acquisition, and the width acquisition between the titanium mesh group and the bone grafting group were compared using the nonparametric independentsamples t-test. Subpopulations within the study groups (exposed vs. not exposed) were compared using the identical test. The width absorption ratio and the width acquisition among three positions (BW, MW, and $\mathrm{CW}$ ) were compared using one-way ANOVA. The reported p-values were two-sided. All calculations were carried out using the GraphPad Prism 8 software. P values $<0.05$ were defined as statistically significant.

\section{Declarations}

\section{Acknowledgements}

Thanks for the patients who participated the follow-up. This work was financially supported by Natural Science Foundation of Fujian Province (2021J01792) and Fujian Province Medical Innovation Project (2021CXA035).

\section{Authors' contributions}

DW and JC contributed to operation of the surgical proceduce. LZ and JMD contributed to the design the surgical proceduce. YFX contributed to the statistical analysis and drafting article. YJL and CWL contributed to date collection and drafting article. All authors read and approved the final manuscript.

\section{Competing interests}

The authors declare no competing interests.

\section{Data Availability}

The datasets used and/or analyzed during the current study are available from the corresponding author on reasonable requested.

\section{Additional information}


The datasets used and/or analyzed during the current study are available from the corresponding author on reasonable requested. The study protocol was evaluated and approved by the Institutional Ethics Committee of the School of Stomatology, Fujian Medical University (Ref. [2019] NO.30). All procedures performed in studies involving human participants were in accordance with the ethical standards of the institutional and/or national research committee and with the 1964 Helsinki declaration and its later amendments or comparable ethical standards. All patients provided written informed consent.

\section{References}

1. Moghadam H. G. Vertical and horizontal bone augmentation with the intraoral autogenous J-graft. Implant Dent. 18, 230-238 (2009).

2. Doonquah L., Lodenquai R., Mitchell A. D. Surgical techniques for augmentation in the horizontally and vertically compromised alveolus. Dental clinics of North America. 59, 389-407 (2015).

3. Nkenke E., Neukam F. W. Autogenous bone harvesting and grafting in advanced jaw resorption: morbidity, resorption and implant survival. European journal of oral implantology. 7 Suppl 2, S203217 (2014).

4. Hameed M. H., Gul M., Ghafoor R., Khan F. R. Vertical Ridge Gain with Various Bone Augmentation Techniques: A Systematic Review and Meta-Analysis. Journal of prosthodontics: official journal of the American College of Prosthodontists. 28, 421-427 (2019).

5. Rh Owen G., Dard M., Larjava H. Hydoxyapatite/beta-tricalcium phosphate biphasic ceramics as regenerative material for the repair of complex bone defects. Journal of biomedical materials research Part B, Applied biomaterials. 106, 2493-2512 (2018).

6. Mendoza-Azpur G., de la Fuente A., Chavez E., Valdivia E., Khouly I. Horizontal ridge augmentation with guided bone regeneration using particulate xenogenic bone substitutes with or without autogenous block grafts: A randomized controlled trial. Clinical implant dentistry and related research. 21, 521-530 (2019).

7. Wessing B., Lettner S., Zechner W. Guided Bone Regeneration with Collagen Membranes and Particulate Graft Materials: A Systematic Review and Meta-Analysis. The International journal of oral \& maxillofacial implants. 33, 87-100 (2018).

8. Zitzmann N. U., Naef R., Schärer P. Resorbable versus nonresorbable membranes in combination with Bio-Oss for guided bone regeneration. The International journal of oral \& maxillofacial implants. 12, 844-852 (1997).

9. Urban I. A., Monje A. Guided Bone Regeneration in Alveolar Bone Reconstruction. Oral Maxillofac Surg Clin North Am. 31, 331-338 (2019).

10. Aprile P., Letourneur D., Simon-Yarza T. Membranes for Guided Bone Regeneration: A Road from Bench to Bedside. Advanced healthcare materials. 9, e2000707 (2020).

11. Rasia-dal Polo M., Poli P. P., Rancitelli D., Beretta M., Maiorana C. Alveolar ridge reconstruction with titanium meshes: a systematic review of the literature. Med Oral Patol Oral Cir Bucal. 19, e639-646 
(2014).

12. Her S., Kang T., Fien M. J. Titanium mesh as an alternative to a membrane for ridge augmentation. Journal of oral and maxillofacial surgery: official journal of the American Association of Oral and Maxillofacial Surgeons. 70, 803-810 (2012).

13. Pourdanesh F., Esmaeelinejad M., Aghdashi F. Clinical outcomes of dental implants after use of tenting for bony augmentation: a systematic review. The British journal of oral \& maxillofacial surgery. 55, 999-1007 (2017).

14. Johar A. O. Ridge Augmentation with Autogenous Bone Graft and Expanded Polytetrafluoroethylene Membrane using Tenting Screw: A Randomized Controlled Clinical Trial. J Contemp Dent Pract. 20, 409-416 (2019).

15. Guillen G. A., et al. Evaluation of the screw tent-pole technique for the repair of anterior maxilla width defects: a prospective, randomized, split-mouth study. International journal of oral and maxillofacial surgery. 50, 801-807 (2021).

16. Block MS, Ducote CW, Mercante DE. Horizontal augmentation of thin maxillary ridge with bovine particulate xenograft is stable during 500 days of follow-up: preliminary results of 12 consecutive patients. Journal of oral and maxillofacial surgery: official journal of the American Association of Oral and Maxillofacial Surgeons. 70, 1321-1330 (2012).

17. Benic G. I., Hämmerle C. H. Horizontal bone augmentation by means of guided bone regeneration. Periodontology 2000. 66, 13-40 (2014).

18. Cordaro L., Torsello F., Miuccio M. T., di Torresanto V. M., Eliopoulos D. Mandibular bone harvesting for alveolar reconstruction and implant placement: subjective and objective cross-sectional evaluation of donor and recipient site up to 4 years. Clinical oral implants research. 22, 1320-1326 (2011).

19. Rakhmatia Y. D., Ayukawa Y., Furuhashi A., Koyano K. Current barrier membranes: titanium mesh and other membranes for guided bone regeneration in dental applications. Journal of prosthodontic research. 57, 3-14 (2013).

20. Block M. S., Ducote C. W., Mercante D. E. Horizontal augmentation of thin maxillary ridge with bovine particulate xenograft is stable during 500 days of follow-up: preliminary results of 12 consecutive patients. Journal of oral and maxillofacial surgery: official journal of the American Association of Oral and Maxillofacial Surgeons. 70, 1321-1330 (2012).

21. Sagheb K., et al. Clinical outcome of alveolar ridge augmentation with individualized CAD-CAMproduced titanium mesh. International journal of implant dentistry. 3, 36 (2017).

22. Raisian S., Fallahi H. R., Khiabani K. S., Heidarizadeh M., Azdoo S. Customized Titanium Mesh Based on the 3D Printed Model vs. Manual Intraoperative Bending of Titanium Mesh for Reconstructing of Orbital Bone Fracture: A Randomized Clinical Trial. Reviews on recent clinical trials. 12, 154-158 (2017).

23. Aloy-Prósper A., Peñarrocha-Oltra D., Peñarrocha-Diago M., Peñarrocha-Diago M. The outcome of intraoral onlay block bone grafts on alveolar ridge augmentations: a systematic review. Med Oral 
Patol Oral Cir Bucal. 20, e251-258 (2015).

24. Briguglio F., et al. The Use of Titanium Mesh in Guided Bone Regeneration: A Systematic Review. International journal of dentistry. 2019, 9065423 (2019).

25. Maiorana C., Santoro F., Rabagliati M., Salina S. Evaluation of the use of iliac cancellous bone and anorganic bovine bone in the reconstruction of the atrophic maxilla with titanium mesh: a clinical and histologic investigation. The International journal of oral \& maxillofacial implants. 16, 427-432 (2001).

26. Xie Y., Li S., Zhang T., Wang C., Cai X. Titanium mesh for bone augmentation in oral implantology: current application and progress. International journal of oral science. 12, 37 (2020).

27. Louis P. J., Gutta R., Said-Al-Naief N., Bartolucci A. A. Reconstruction of the maxilla and mandible with particulate bone graft and titanium mesh for implant placement. Journal of oral and maxillofacial surgery: official journal of the American Association of Oral and Maxillofacial Surgeons. 66, 235-245 (2008).

28. Cucchi A., Vignudelli E., Napolitano A., Marchetti C., Corinaldesi G. Evaluation of complication rates and vertical bone gain after guided bone regeneration with non-resorbable membranes versus titanium meshes and resorbable membranes. A randomized clinical trial. Clinical implant dentistry and related research. 19, 821-832 (2017).

29. Atef M., Tarek A., Shaheen M., Alarawi R. M., Askar N. Horizontal ridge augmentation using native collagen membrane vs titanium mesh in atrophic maxillary ridges: Randomized clinical trial. Clinical implant dentistry and related research. 22, 156-166 (2020).

30. Urban I. A., Monje A., Lozada J., Wang H. L. Principles for Vertical Ridge Augmentation in the Atrophic Posterior Mandible: A Technical Review. The International journal of periodontics \& restorative dentistry. 37, 639-645 (2017).

\section{Tables}

Table 1 the basic information of patients in different groups.

\begin{tabular}{|llc|}
\hline Mean range & $\begin{array}{l}\text { Titanium } \\
\text { mesh }\end{array}$ & $\begin{array}{l}\text { autologous } \\
\text { bone }\end{array}$ \\
\hline Male/female & $3 / 5$ & $3 / 4$ \\
\hline age & $26.85 \pm 4.83$ & $30.55 \pm 13.04$ \\
\hline $\begin{array}{l}\text { the region of bone defect: anterior region of } \\
\text { maxillary/mandible }\end{array}$ & $813 / 0$ & $57 / 23$ \\
\hline smoking & 0 & 0 \\
\hline periodontal disease & 0 & 0 \\
\hline gingival biotype: thick/thin & $6 / 3$ & $5 / 2$ \\
\hline
\end{tabular}


Table 2 Gain bone width after 6 months of surgery of different groups.

\begin{tabular}{|lllll|}
\hline Gain bone width & onlay grafting & \multicolumn{3}{l|}{ Titanium mesh } \\
\cline { 3 - 5 } & & Total & Not exposure & exposure \\
\hline crestal width & $3.458 \pm 0.878$ & $4.683 \pm 2.357$ & $5.214 \pm 2.609$ & $3.940 \pm 1.971$ \\
\hline midway width & $3.494 \pm 0.570$ & $4.383 \pm 2.336$ & $5.157 \pm 2.461$ & $3.300 \pm 1.867$ \\
\hline basal width & $3.337 \pm 0.882$ & $4.575 \pm 1.876$ & $5.114 \pm 2.113$ & $3.820 \pm 1.325$ \\
\hline
\end{tabular}

\section{Figures}
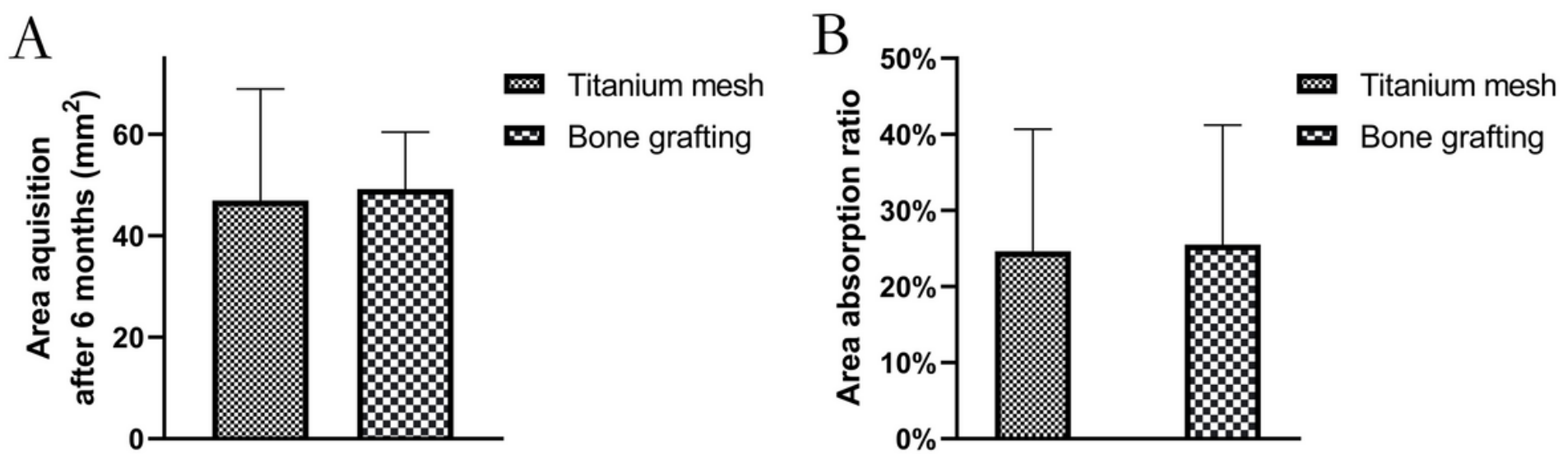

Figure 1

The area acquisition (A) and the absorption ratio (B) of the two studied groups.
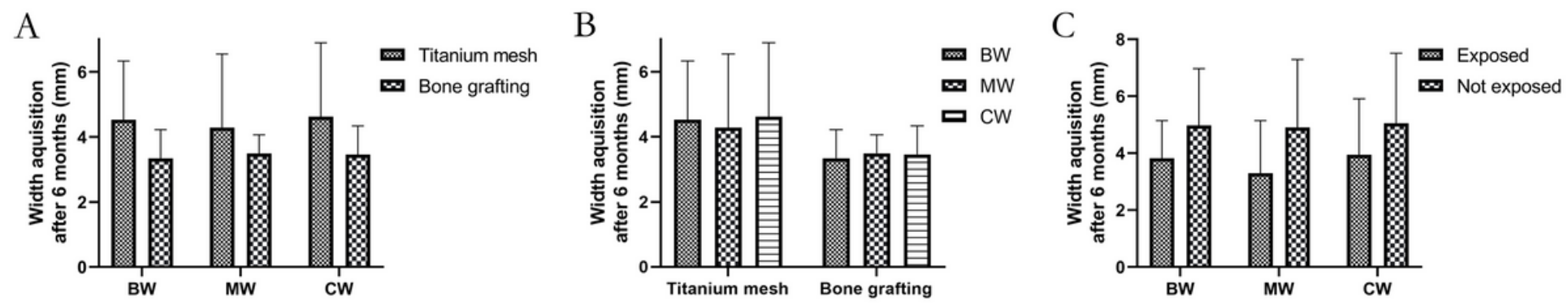

Figure 2

The gained bone width after 6 months of surgery in two studied groups at different measured level (A), at three different level of the two studied groups (B), and in the exposed or not exposed one at different measured level (C). 
A

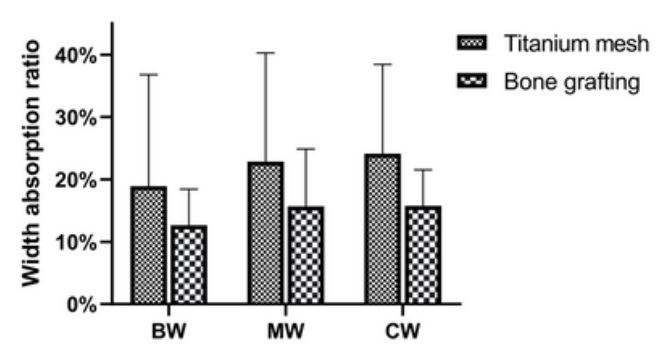

B

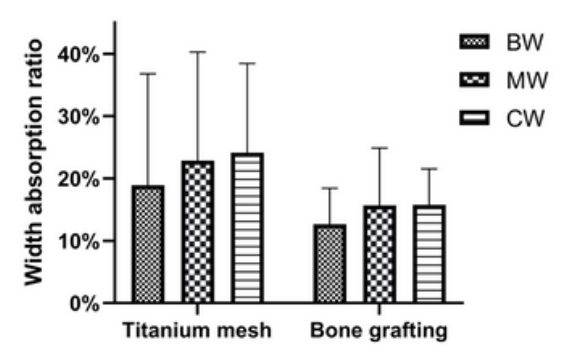

C

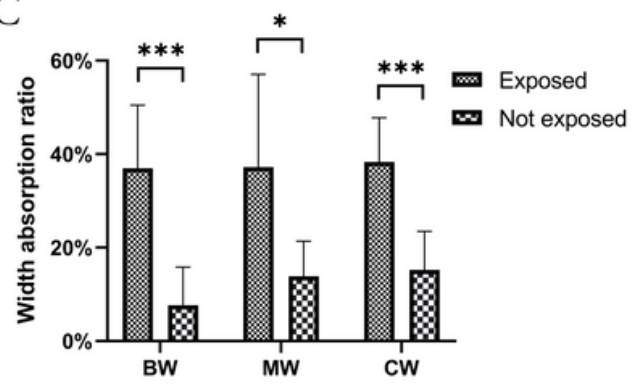

\section{Figure 3}

The width absorption ratio in two studied groups at different measured level (A), at three different level of the two studied groups (B), and in the exposed or not exposed one at different measured level (C). ${ }^{*} \mathrm{p}$ $0.05, * * * p<0.001$

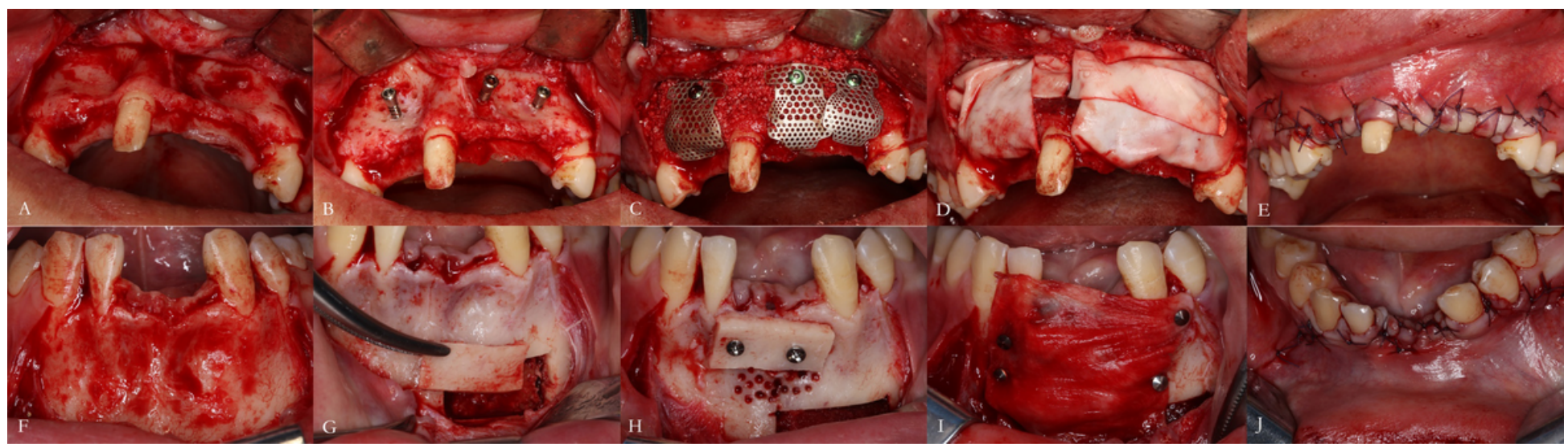

\section{Figure 4}

The briefly process of bone augmentation using titanium mesh with tenting screw and autogenous bone block. A: Atrophy ridge present after full-thickness mucoperiosteal flap was reflected. B: Tenting screws were fixed onto the alveolar ridge. C: Titanium mesh was fixed onto the tenting screw. D: Covering absorbable membranes. E: The flap was repositioned and sutured. F: Atrophy ridge present after fullthickness mucoperiosteal flap was reflected. G: The autologous bone was collected from chin. $\mathrm{H}$ : The autologous bone block was fixed on the alveolar ridge. I: Covering absorbable membranes. J: The flap was repositioned and sutured 


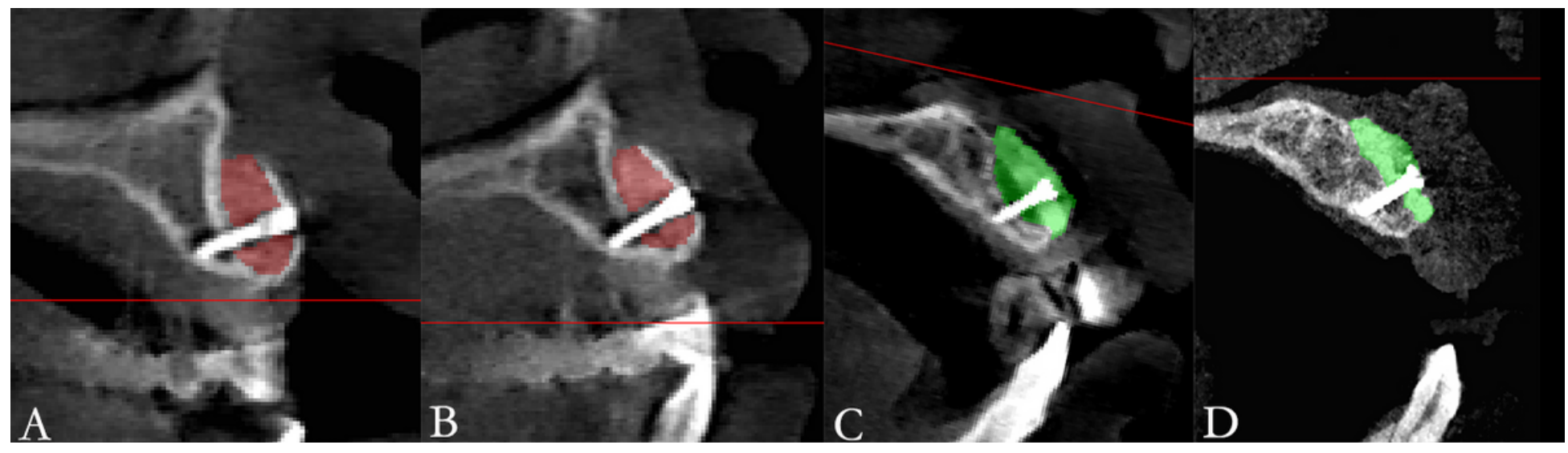

\section{Figure 5}

The area measurement of the cross section of the tenting screw in titanium mesh group (A: immediately after the surgery and B: 6 months after the surgery) and titanium screw in onlay grafting group (C: immediately after the surgery and D: 6 months after the surgery).

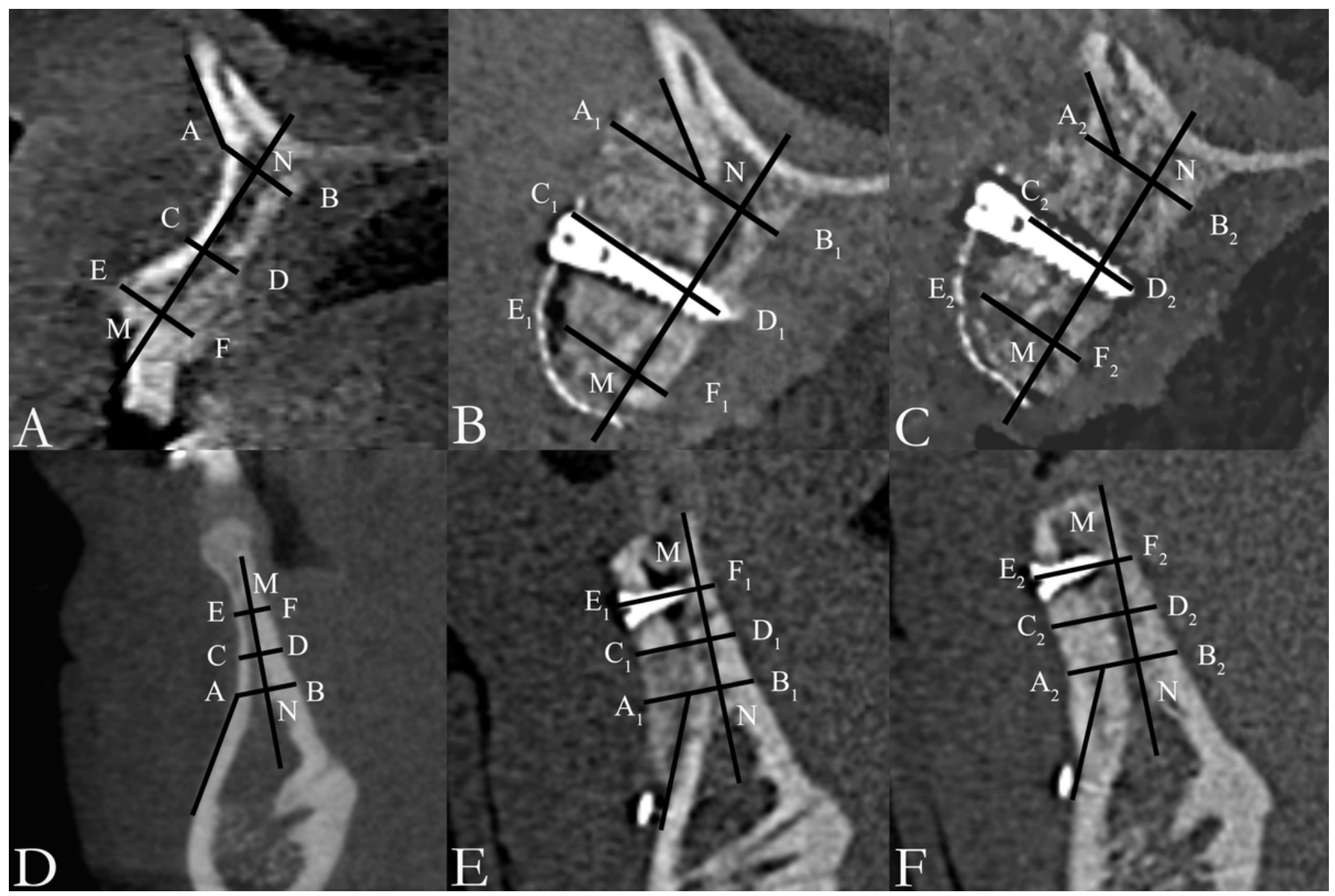

Figure 6 
The measured points at different level of cross section of the tenting screw in titanium mesh group (A: before surgery, $B$ : immediately after the surgery and C: 6 months after the surgery) and titanium screw in onlay grafting group (D: before surgery, E: immediately after the surgery and F: 6 months after the surgery).

\section{Supplementary Files}

This is a list of supplementary files associated with this preprint. Click to download.

- supplementaryfile1.xlsx

- supplementaryfile2.xlsx 